\title{
Estratégias para modificar hábitos de sono, alimentação e atividade física no combate à obesidade infantil
}

\author{
Strategies for modifying sleeping, eating, and physical \\ activity habits for combating childhood obesity
}

\author{
Mariana Bezerra Rocha Figueiredo ${ }^{1}$, Márcio Moysés de Oliveira², \\ Cristiane Fiqueni Conti ${ }^{2}$, João Eduardo Coin-Carvalho ${ }^{3}$, Lucila \\ Bizari Fernandes do Prado ${ }^{4}$, Gilmar Fernandes do Prado ${ }^{4}$, \\ Luciane Bizari Coin de Carvalho ${ }^{5}$
}

\begin{abstract}
1.Psicóloga, Doutora, Neuro-Sono, Departamento de Medicina, Unifesp, São Paulo-SP, Brasil.
2.Médico, Doutor, Universidade Federal do Maranhão - UFMA, São Luis-MA, Brasil.

3.Psicólogo, Doutor, Universidade Paulista - UNIP, São Paulo-SP, Brasil.

4.Médico, Doutor, Neuro-Sono, Departamento de Neurologia e Neurocirurgia, Unifesp, São Paulo-SP, Brasil.

5.Psicóloga, Doutora, Neuro-Sono, Departamento de Neurologia e Neurocirurgia, Unifesp, São Paulo-SP, Brasil.
\end{abstract}

\begin{abstract}
Resumo
Introdução. A obesidade é um problema de saúde pública e, portanto, a intervenção para reduzir essa epidemia se torna uma necessidade primordial. O objetivo deste estudo foi determinar a eficácia de estratégias para modificar hábitos de sono, alimentação e atividade física no combate à obesidade infantil em crianças em idade escolar. Método. Trata-se de uma revisão sistemática de estudos randomizados, com crianças de 6 a 10 anos, sobre intervenções para redução de peso, melhora da atividade física e sono, com os desfechos de Índice de Massa Corporal (IMC) e circunferência da cintura (CC). Resultados. Sete artigos foram incluídos. O programa Girls' health Enrichment Multi-site Studies (GEMS) - 12 semanas apresentou: o IMC $(p=0,28)$ e a CC $(p<0,001)$ foram menores para o grupo intervenção em relação ao grupo controle. O programa de Intervenção Comportamental apresentou: IMC menor $(p=0,44)$ para o grupo intervenção em relação ao grupo controle. $O$ GEMS - 24 meses não apresentou diferenças entre os grupos. Os desfechos apresentaram heterogeneidade devido à amostra pequena e viés metodológico. Conclusão. O Programa GEMS - 12 semanas possibilitou diminuição da Circunferência da Cintura, com uma fraca qualidade de evidência. Há necessidade de mais ensaios clínicos randomizados para estratégias escolares de prevenção da obesidade, com base nas necessidades das crianças, incluindo a família e o sono, com ampliação dos desfechos estudados e tamanho da amostra. Unitermos. obesidade; atividade física; sono; nutrição; revisão sistemática
\end{abstract}

\begin{abstract}
Introduction. Obesity is a public health problem and therefore, intervention to reduce this epidemic becomes a prime necessity. The objective of this study was to determine the effectiveness of strategies for modifying sleeping, eating, and physical activity habits for combating childhood obesity among school-age children. Method. This was a systematic review of randomized trials, with children aged 6-10yo, about interventions to reduce weight, improve physical activity, and sleep; and for outcomes body mass index (BMI) and waist circumference (WC). Results. Seven papers were included. The program: Girls' health Enrichment Multi-site Studies (GEMS) - 12 weeks resulted reduction of BMI $(p=0.28)$ and WC $(p<0.001)$ for intervention group compared to control group. The program: Behavioral Interventions resulted reduction of BMI $(p=0.44)$ for intervention group. The program: GEMS - 24 months did not result differences between groups. The outcomes presented heterogeneity because small sample and methodological bias. Conclusion. The GEMS program - 12 weeks reduced WC with a weak quality of evidence. There is a need for randomized controlled trials evaluating school-based strategies for obesity prevention based on children necessities, including the family and sleep, with adequate samples and outcomes.
\end{abstract}


Trabalho realizado na Escola Paulista de Medicina (EPM) da Universidade Federal de São Paulo (Unifesp), São Paulo-SP, Brasil.

\section{INTRODUÇÃO}

Uma tendência ao aumento da prevalência de sobrepeso e obesidade em crianças em idade escolar, préescolar e adolescentes tem sido observada em todo o mundo ${ }^{1}$. Em um estudo com 1.616 crianças e adolescentes, a prevalência de sobrepeso foi de $22,2 \%$ em crianças préescolares, $12,9 \%$ em idade escolar e $10,8 \%$ em adolescentes, enquanto a obesidade foi detectada em $13,8 \%$ dos pré-escolares, 8,2\% em idade escolar, e 4,9\% em adolescentes ${ }^{2}$.

Estudos recentes sugeriram que reduções no tempo habitual de sono e os transtornos do sono podem complementar a gama de causalidade da obesidade infantil ${ }^{1,3}$.

O transtorno respiratório do sono (TRS) é uma condição comum e está associada a anormalidades metabólicas, incluindo diabetes, hipertensão e obesidade ${ }^{4,5}$. Estudos anteriores documentaram a associação entre uma variedade de marcadores para TRS (incluindo ronco, sonolência diurna ou duração do sono) e a síndrome metabólica ou seus componentes na população em geral ${ }^{4}$.

A obesidade ou sobrepeso e a apneia obstrutiva do sono (AOS) estão bem correlacionados em adultos, 
adolescentes e, em menor grau, em crianças pequenas. Evidências sugerem que o excesso de peso pode causar a Síndrome da Apneia Obstrutiva do Sono (SAOS). No entanto, a SAOS também pode manter o excesso de peso por meio de mecanismos ligados a disfunções metabólicas que podem contribuir para o ganho de peso e também para o sono não reparador. O sono inadequado pode causar desatenção, impulsividade, humor negativo e disfunção cognitiva, além de alterar os níveis de leptina e grelina relacionados ao apetite, contribuindo para más escolhas alimentares, como alimentos doces, ricos em calorias e ricos em amido6-9. Pode ser particularmente importante entender a relação entre AOS e obesidade do meio ao final da infância, quando as escolhas alimentares são cada vez mais autodeterminadas e 0 peso se torna altamente preditivo da obesidade adulta e de sua morbidade ${ }^{7,8}$.

A duração do sono é determinada por vários fatores. Alguns deles podem ser difíceis de controlar, enquanto outros, como hora de dormir, hora de acordar, ajuste da fase do sono aos ritmos circadianos subjacentes, compromissos obrigatórios (por exemplo, dias de escola versus dias de final de semana), tempo de sono (reduzido ou fragmentado) e transtornos respiratórios do sono (síndrome da apneia obstrutiva do sono ou ronco) pode ser modificada ${ }^{10,11}$.

Evidências de outros estudos, incluindo uma metanálise relacionada ao sono curto e obesidade entre 
adultos e crianças, sugerem que o aumento do tempo de sono pode diminuir a atual epidemia de obesidade ${ }^{12}$.

Para crianças, a prevenção e o controle da obesidade associados à duração do sono não foram explorados extensivamente. Além disso, e mais importante, as avaliações se basearam principalmente em estimativas subjetivas da duração do sono ${ }^{11}$.

Além disso, causas multifatoriais, incluindo causas genéticas, ambientais, sociais e econômicas, alterações nos hábitos alimentares, diminuição dos níveis de atividade física e falta de informações adequadas para os pais, contribuem para o aumento da prevalência de obesidade ${ }^{1,13-16}$.

Alguns estudos demonstraram que horas excessivas em frente à TV, computador e videogame, falta de programas de educação física nas escolas e o estilo de vida sedentário dos pais são fatores agravantes ${ }^{16-19}$.

A obesidade é um problema de saúde pública e, portanto, a intervenção para reduzir essa epidemia se torna uma necessidade primordial ${ }^{20}$. Para incluir o sono em uma estratégia abrangente de prevenção do excesso de peso, são necessários estudos de intervenção que analisem o efeito potencialmente protetor da duração prolongada do sono. Assim, este estudo teve como objetivo determinar a eficiência de estratégias de prevenção e controle da obesidade em crianças em idade escolar, incluindo o estudo de mudanças na duração do sono, utilizando a revisão sistemática. 


\section{MÉTODO}

Realizamos uma pesquisa eletrônica nas bases de dados Cochrane Library, Pubmed, Lilacs e Embase, de ensaios clínicos randomizados, atualizados em 2016, utilizando os seguintes termos e seus sinônimos: obesidade, exercício, atividade motora, hábitos alimentares, comportamento alimentar, dieta, sono, distúrbios do sono, síndrome da apneia obstrutiva do sono, desenvolvimento de programas, promoção da saúde, nutrição. Além disso, outras fontes também foram consideradas: as indexadas na BIREME/OPAS-OMS (Biblioteca Regional de Medicina/Organização PanAmericana da Saúde da Organização Mundial da Saúde); listas de referência de todos os ensaios clínicos recuperados; informações adicionais solicitadas aos autores dos estudos primários por meio de comunicação por correio eletrônico.

\section{Participantes}

Estudos com crianças de 6 a 10 anos, com variáveis relacionadas ao peso, incluindo uma medida de controle, alterações na ingestão de alimentos e alterações na duração total do sono. Tipos de intervenções: Todas as intervenções para prevenção e controle da obesidade relacionadas à nutrição, atividade física, comportamento do sono e redução no tempo de televisão ou combinações dos mesmos. Grupos de comparação: placebo, sem intervenção e outras intervenções não direcionadas à prevenção da 
obesidade. Desfecho: diminuição do índice de massa corporal (IMC) e diminuição da circunferência da cintura (CC). Desfechos secundários: aumento da duração total do sono, aumento da qualidade do sono, diminuição do índice de apneia, aumento da qualidade de vida, alterações na ingestão de alimentos, diminuição da ingestão de alimentos sem qualidade e aumento da ingestão de leite e vegetais; e aumento dos níveis de atividade física.

\section{Seleção dos estudos}

A revisão foi realizada por dois autores que examinaram independentemente os estudos e extraíram os dados usando formulários padrão de extração de dados. Discordâncias e discrepâncias foram resolvidas em consulta com um terceiro autor da revisão.

\section{Qualidade metodológica dos estudos incluídos}

Os estudos foram julgados de acordo com a metodologia do manual Cochrane, com base na ocultação de alocação (randomização), cegamento (viés de desempenho e detecção) e viés de atrito. Os estudos incluídos também foram considerados como risco baixo, alto ou pouco claro de viés.

\section{Análise dos dados}

A síntese e análise dos dados foram realizadas no software Cochrane Review Manager (RevMan), versão 4.2.8. A diferença da média ponderada ( $A D M$ ) foi usada 
quando dados contínuos estavam disponíveis nos estudos incluídos, com intervalos de confiança de 95\% (IC). A análise estatística usando um modelo de efeito fixo ${ }^{21}$ foi usada para estimar os efeitos do tratamento em comparação com o grupo controle. A significância estatística foi considerada quando $p<0,05$. A heterogeneidade entre os efeitos estimados em diferentes estudos foi analisada pelo teste de inconsistência $\left(\mathrm{I}^{2}\right)$, que descreve a porcentagem da variabilidade nas estimativas de efeito que é devida à heterogeneidade mais do que ao erro amostral ${ }^{22}$. A heterogeneidade estatística foi assumida nos casos em que $\mathrm{I}^{2}>50 \%$. De acordo com o tipo de informação disponível nos artigos ou obtida entrando em contato com os autores dos estudos primários correspondentes, os resultados foram agrupados com base nas alterações da linha de base e nas subcategorias de duração da intervenção.

\section{RESULTADOS}

Foram identificados 96 ensaios publicados (em inglês, alemão, francês, português e espanhol) relacionados à prevenção e controle da obesidade em crianças em idade escolar. Sete ensaios clínicos randomizados (todos em inglês) foram incluídos nesta revisão, uma vez que preenchiam os critérios de inclusão (Tabela 1) ${ }^{23-29}$. Oitenta e cinco ensaios foram excluídos: 2 incluíram crianças desde o nascimento, 57 incluíram crianças entre 2 e 18 anos sem 
análise de dados específicos e 30 foram ensaios clínicos sem resultados relacionados ao peso.

Um total de 650 participantes foi randomizado em estudos de desenho paralelo. Esses participantes receberam intervenções diferentes em comparação com o grupo controle.

O programa de intervenção dos 7 artigos foi baseado em diretrizes sobre atividade física e alimentação saudável. Apenas um artigo usou aulas de dança depois da escola como parte do programa 25 .

Várias medidas subjetivas (questionários/entrevistas específicos) e medidas objetivas (resultados relacionados ao peso) foram usadas nesses estudos; os efeitos estimados do tratamento versus controle foram analisados de acordo com os seguintes resultados: índice de massa corporal (IMC) e circunferência da cintura (CC) e, quando analisados por subgrupos, conseguimos realizar a metanálise. 
Tabela 1. Características dos estudos incluídos nesta revisão.

\begin{tabular}{|c|c|c|c|c|}
\hline Estudo & Método & Participantes & Intervenções & Variáveis \\
\hline $\begin{array}{l}\text { Baranowski } \\
2003^{23}\end{array}$ & $\begin{array}{l}\text { Estudo Piloto } \\
\text { Randomizado } \\
\text { Controlado } \\
\text { Paralelo }\end{array}$ & $\begin{array}{l}\mathrm{n}=35 \text { + } \\
\text { idade: } 8 \text { anos } \\
\text { GI: } 19 \\
\text { GC: } 16\end{array}$ & $\begin{array}{l}12 \text { semanas } \\
\text { Orientação para pais } \\
\text { e filhos sobre } \\
\text { mudanças nos } \\
\text { hábitos de } \\
\text { alimentação } \\
\text { saudável e atividade } \\
\text { física. } \\
\text { GI: } 4 \text { semanas no } \\
\text { acampamento de } \\
\text { verão e internet } \\
\text { semanalmente } \\
\text { durante } 8 \text { semanas } \\
\text { em casa. } \\
\text { GC: } 4 \text { semanas em } \\
\text { acampamento de } \\
\text { verão (outras } \\
\text { atividades) e internet } \\
\text { mensalmente por } 8 \\
\text { semanas em casa. }\end{array}$ & $\begin{array}{l}\text { SB]: Consumo } \\
\text { de frutas, } \\
\text { verduras e } \\
\text { atividade } \\
\text { física. } \\
\text { OBJ: IMC e CC }\end{array}$ \\
\hline $\begin{array}{l}\text { Beech } \\
2003^{24}\end{array}$ & $\begin{array}{l}\text { Estudo Piloto } \\
\text { Randomizado } \\
\text { Controlado } \\
\text { Paralelo }\end{array}$ & $\begin{array}{l}\mathrm{n}=60 \\
\text { idade: } 8-10 \\
\text { anos } \\
\text { GI: } 21 \text { crianças } \\
\text { e } 21 \text { pais } \\
\text { GC: } 18\end{array}$ & $\begin{array}{l}12 \text { semanas } \\
\text { Orientação para pais } \\
\text { e filhos sobre } \\
\text { mudanças nos } \\
\text { hábitos de } \\
\text { alimentação } \\
\text { saudável e atividade } \\
\text { física. } \\
\text { GI: orientação } \\
\text { semanal sobre } \\
\text { hábitos alimentares } \\
\text { saudáveis e } \\
\text { atividade física. } \\
\text { GC: orientação } \\
\text { mensal sobre hábitos } \\
\text { alimentares } \\
\text { saudáveis e } \\
\text { atividade física. }\end{array}$ & $\begin{array}{l}\text { SBJ: Consumo } \\
\text { de bebidas } \\
\text { açucaradas e } \\
\text { água. } \\
\text { OBJ: IMC, CC, } \\
\text { composição } \\
\text { corporal e } \\
\text { atividade física } \\
\text { por } \\
\text { acelerômetro. }\end{array}$ \\
\hline $\begin{array}{l}\text { Robinson } \\
2003^{25}\end{array}$ & $\begin{array}{l}\text { Estudo } \\
\text { Randomizado } \\
\text { Controlado } \\
\text { Paralelo }\end{array}$ & $\begin{array}{l}\mathrm{n}=61+ \\
\text { idade: } 8-10 \\
\text { anos } \\
\text { perda: } 09 \\
\text { GI: } 25 \\
\text { GC: } 27\end{array}$ & $\begin{array}{l}12 \text { semanas } \\
\text { IG: orientação para } \\
\text { a prática diária de } \\
\text { aulas de dança, } \\
\text { mudança de hábitos } \\
\text { alimentares e } 5 \\
\text { aulas ministradas em } \\
\text { casa com instruções } \\
\text { para reduzir o tempo } \\
\text { assistindo TV, vídeo } \\
\text { e videogame. } \\
\text { GC: orientações } \\
\text { mensais sobre dieta } \\
\text { e } 5 \text { aulas ministradas } \\
\text { em casa, com } \\
\text { orientações sobre os } \\
\text { riscos da obesidade. }\end{array}$ & $\begin{array}{l}\text { SBJ: uso da } \\
\text { mídia } \\
\text { autorreferida } \\
\text { com refeições } \\
\text { vendo } \\
\text { televisão. } \\
\text { OBJ: IMC, CC e } \\
\text { atividade física } \\
\text { por } \\
\text { acelerômetro }\end{array}$ \\
\hline
\end{tabular}

GI: grupo intervenção; GC: grupo controle; SBJ: subjetivo; OBJ: objetivo; IMC: índice de massa corporal; CC: circunferência da cintura. 
Tabela 1 (cont.). Características dos estudos incluídos nesta revisão.

\begin{tabular}{|c|c|c|c|c|}
\hline $\begin{array}{l}\text { Story } \\
2003^{26}\end{array}$ & $\begin{array}{l}\text { Estudo Piloto } \\
\text { Randomizado } \\
\text { Controlado } \\
\text { Paralelo }\end{array}$ & $\begin{array}{l}\mathrm{n}=54+ \\
\text { idade: } 8-10 \\
\text { anos } \\
\text { GI: } 26 \\
\text { GC: } 28\end{array}$ & $\begin{array}{l}12 \text { semanas } \\
\text { GI: após as } \\
\text { atividades escolares, } \\
\text { duas vezes por } \\
\text { semana para } \\
\text { crianças e pais, com } \\
\text { foco no aumento da } \\
\text { atividade física e } \\
\text { alimentação } \\
\text { saudável. } \\
\text { GC: recebeu um } \\
\text { programa não } \\
\text { relacionado à } \\
\text { nutrição ou atividade } \\
\text { física, participou de } \\
\text { reuniões mensais (3 } \\
\text { reuniões) para } \\
\text { melhorar a auto- } \\
\text { estima e o } \\
\text { conhecimento } \\
\text { cultural. }\end{array}$ & $\begin{array}{l}\text { SBJ: variáveis } \\
\text { autorreferida } \\
\text { psicossociais e } \\
\text { demográficas, } \\
\text { atividade } \\
\text { física. } \\
\text { OBJ: IMC, CC, } \\
\text { composição } \\
\text { corporal e } \\
\text { atividade física } \\
\text { por } \\
\text { acelerômetro. }\end{array}$ \\
\hline $\begin{array}{l}\text { Kalavainen } \\
2007^{27}\end{array}$ & $\begin{array}{l}\text { Estudo } \\
\text { Randomizado } \\
\text { Controlado } \\
\text { Paralelo }\end{array}$ & $\begin{array}{l}\mathrm{n}=70 \\
\hat{\jmath}=28 \\
\text { o=42 } \\
\text { perda: } 01 \\
\text { idade: } 7-9 \\
\text { anos }\end{array}$ & $\begin{array}{l}1 \text { ano ( } 6 \text { meses mais } \\
6 \text { meses de } \\
\text { acompanhamento) } \\
\text { GI: } 15 \text { sessões } \\
\text { durante } 6 \text { meses (10 } \\
\text { semanais e } 5 \\
\text { quinzenais), durante } \\
90 \text { minutos para pais } \\
\text { e filhos sobre } \\
\text { atividade física e } \\
\text { educação nutricional. } \\
\text { GC: } 2 \text { sessões ao } \\
\text { longo de } 6 \text { meses } \\
\text { (reuniões individuais } \\
\text { com a criança), } \\
\text { durante } 30 \text { minutos, } \\
\text { sobre atividade física } \\
\text { e educação } \\
\text { nutricional. }\end{array}$ & OBJ: IMC \\
\hline $\begin{array}{l}\text { Chen } \\
2010^{28}\end{array}$ & $\begin{array}{l}\text { Estudo } \\
\text { Randomizado } \\
\text { Controlado } \\
\text { Paralelo }\end{array}$ & $\begin{array}{l}\mathrm{n}=67 \\
\text { idade: } 8-10 \\
\text { anos }\end{array}$ & $\begin{array}{l}\text { GI: sessões } \\
\text { semanais durante } 8 \\
\text { semanas, } 45 \\
\text { minutos ( } 15 \text { minutos } \\
\text { de atividade física e } \\
30 \text { minutos de } \\
\text { orientação } \\
\text { educacional) para } \\
\text { crianças e duas } \\
\text { sessões durante oito } \\
\text { semanas, com } \\
\text { duração de } 2 \text { horas } \\
\text { de aconselhamento } \\
\text { nutricional e hábitos } \\
\text { saudáveis para } \\
\text { prevenir a obesidade } \\
\text { dos pais . } \\
\text { CG: lista de espera. }\end{array}$ & $\begin{array}{l}\text { SBJ: Consumo } \\
\text { de frutas, } \\
\text { verduras, } \\
\text { conhecimento } \\
\text { nutricional e } \\
\text { atividade } \\
\text { física. } \\
\text { OBJ: IMC, } \\
\text { razão cintura / } \\
\text { rasgo, pressão } \\
\text { arterial e } \\
\text { computador de } \\
\text { atividades } \\
\text { pessoais } \\
\text { Caltrac. }\end{array}$ \\
\hline
\end{tabular}

GI: grupo intervenção; GC: grupo controle; SBJ: subjetivo; OBJ: objetivo; IMC: índice de massa corporal; CC: circunferência da cintura. 
Tabela 1 (cont.). Características dos estudos incluídos nesta revisão.

\begin{tabular}{|c|c|c|c|c|}
\hline $\begin{array}{l}\text { Klesges } \\
2010^{29}\end{array}$ & $\begin{array}{l}\text { Estudo } \\
\text { Randomizado } \\
\text { Controlado } \\
\text { Paralelo }\end{array}$ & $\begin{array}{l}\mathrm{n}=303 \text { + } \\
\text { idade: } 8-10 \\
\text { anos }\end{array}$ & $\begin{array}{l}\text { GI: } 34 \text { sessões ao } \\
\text { longo de } 24 \text { meses } \\
\text { (14 semanais, } 20 \\
\text { mensais), durante } \\
90 \text { minutos, sobre } \\
\text { atividade física e } \\
\text { educação nutricional. } \\
\text { GC: } 34 \text { sessões ao } \\
\text { longo de } 24 \text { meses } \\
\text { (14 semanais, } 20 \\
\text { mensais), durante } \\
90 \text { minutos, sobre } \\
\text { autoestima e } \\
\text { desempenho social. }\end{array}$ & $\begin{array}{l}\text { SBJ: Consumo } \\
\text { de bebidas } \\
\text { açucaradas e } \\
\text { água. } \\
\text { OBJ: IMC e } \\
\text { atividade física } \\
\text { por } \\
\text { acelerômetro. }\end{array}$ \\
\hline
\end{tabular}
corporal; CC: circunferência da cintura.

1.Girls health Enrichment Multi-site Studies (GEMS) - 12s: 210 participantes foram incluídos no GEMS ou grupo controle, durante 12 semanas, com desenho paralelo, nos estudos de Baranowski ${ }^{23}$, Beech.24, Robinson.24, Robinson.25 e Story ${ }^{26}$, possibilitando 2 metanálises (Figuras 1 e 2): a) 0 grupo GEMS reduziu o IMC em -0,04 pontos (IC95\%: -0,13 a 0 ,04) mais do que o grupo controle, com diferença não significante $(p=0,28)$; e diminuiu a CC em $-0,82$ pontos (IC 95\%: 0,61 a 1,03) a mais que o grupo GEMS ( $p$ $<0,00001)$. As variáveis apresentaram grande heterogeneidade.

2.Girls health Enrichment Multi-site Studies (GEMS) - 24m: 303 participantes foram incluídos no GEMS ou grupo controle, durante 24 meses, com desenho paralelo, no estudo de Klesges ${ }^{29}$. Não houve diferenças entre os dois grupos quanto ao IMC ou quaisquer outras medidas antropométricas nas medidas de 1 e 2 anos. 
Figura 1. Resultados do Índice de Massa Corporal (BMI), comparando Girls health Enrichment Multi-site Studies (GEMS) - 12s e grupo controle.

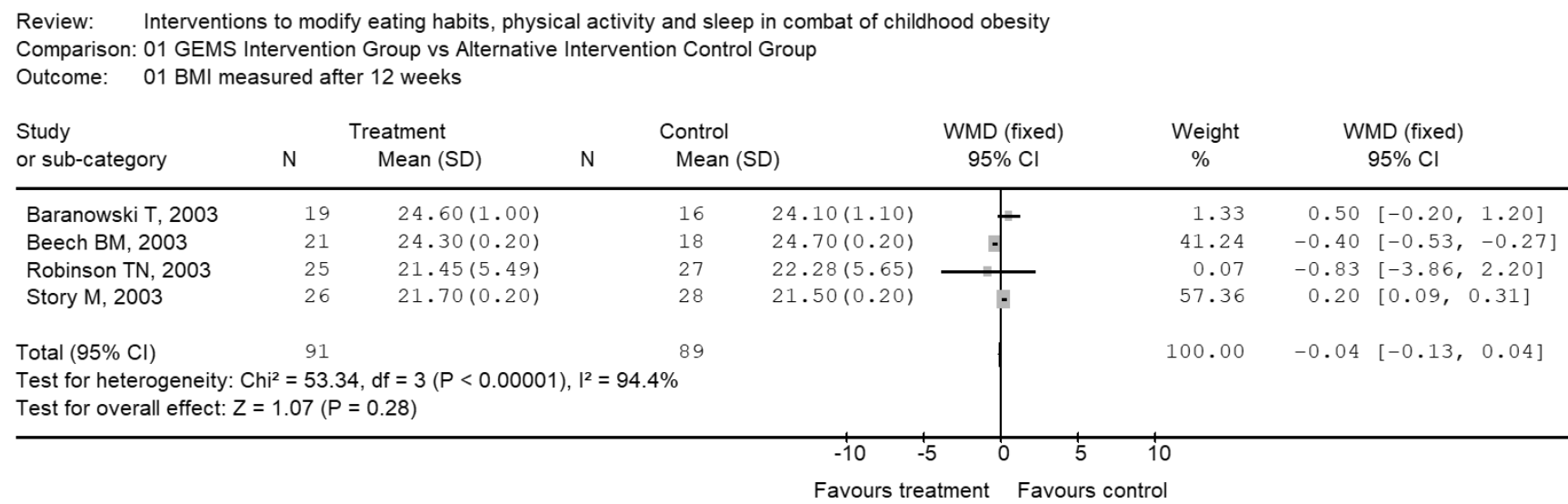

Mean = media $; \mathrm{SD}=$ desvio padrão $; \mathrm{WMD}=$ diferença media ponderada $; \mathrm{CI}=$ interval de confiância .

Figura 2. Resultados da Circunferência da Cintura (WC), comparando Girls health Enrichment Multi-site Studies (GEMS) - 12s e grupo controle.

Review: Interventions to modify eating habits, physical activity and sleep in combat of childhood obesity

Comparison: 02 GEMS Intervention Group vs Alternative Intervention Control Group

Outcome: $\quad 01 \mathrm{WC}$ measured after 12 weeks

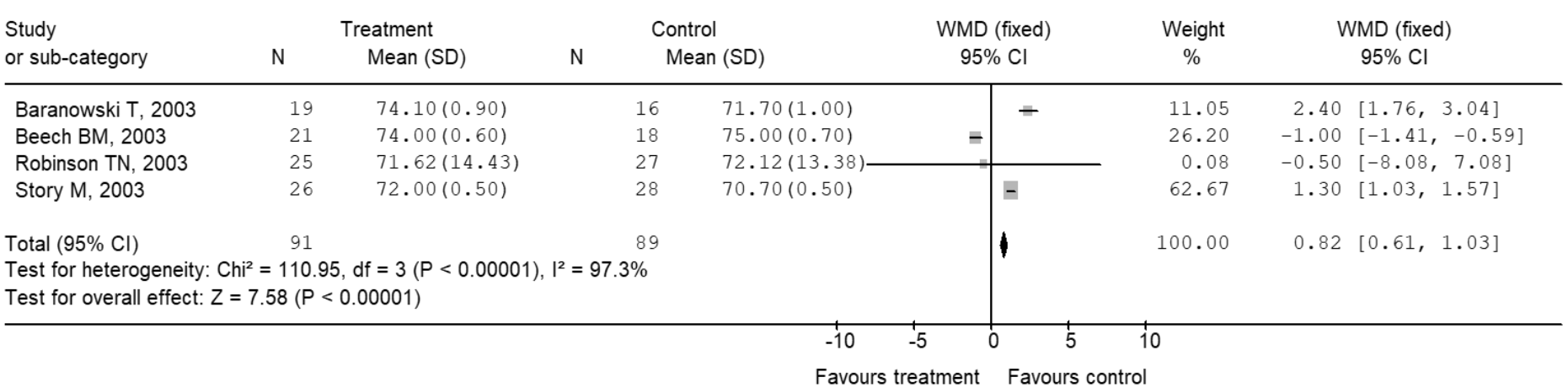

Mean $=$ media $; \mathrm{SD}=$ desvio padrão $; \mathrm{WMD}=$ diferença media ponderada $; \mathrm{CI}=$ interval de confiância .

3. Intervenção Comportamental: 137 participantes foram incluídos em programa comportamental ou intervenção alternativa, durante 6 meses, com desenho paralelo, nos estudos de Kalavainen ${ }^{27}$ e Chen $^{28}$, com medidas de IMC 
(Tabela 1), possibilitando metanálise (Figura 3): a) o grupo Programa de Comportamento reduziu o IMC em -0,18 pontos (IC $95 \%$ : $-0,65$ a 0,28 ) a mais que o grupo controle $(p=0,44)$ com diferença não significante e grande heterogeneidade.

Figura 3. Resultados do Índice de Massa Corporal (BMI), comparando Intervenção Comportamental e Intervenção Alternativa.

Review: Interventions to modify eating habits, physical activity and sleep in combat of childhood obesity Comparison:03 Behavioral Intervention vs Alternative Intervention Control Group

Outcome: $01 \mathrm{BMI}$ measured after 12 weeks

\begin{tabular}{|c|c|c|c|c|c|c|c|c|c|c|}
\hline \multirow{2}{*}{$\begin{array}{l}\begin{array}{l}\text { Study } \\
\text { or sub-category }\end{array} \\
\text { Kalavainen MP, } 2007\end{array}$} & \multicolumn{2}{|c|}{ Treatment } & \multicolumn{3}{|c|}{ Control } & $\begin{array}{c}\text { WMD (fixed) } \\
95 \% \mathrm{Cl}\end{array}$ & $\begin{array}{c}\text { Weight } \\
\%\end{array}$ & \multicolumn{3}{|c|}{$\begin{array}{l}\text { WMD (fixed) } \\
95 \% \mathrm{Cl}\end{array}$} \\
\hline & 35 & $22.60(1.00)$ & & 35 & $22.90(1.10)$ & - & 89.97 & -0.30 & {$[-0.79$,} & $0.19]$ \\
\hline Chen JL, 2010 & 35 & $19.29(3.45)$ & & 32 & $18.42(2.69)$ & 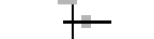 & 10.03 & 0.87 & {$[-0.60$,} & $2.34]$ \\
\hline Total $(95 \% \mathrm{Cl})$ & 70 & & & 67 & & 1 & 100.00 & -0.18 & {$[-0.65$,} & $0.28]$ \\
\hline \multicolumn{11}{|c|}{$\begin{array}{l}\text { Test for heterogeneity: } \mathrm{Chi}^{2}=2.18, \mathrm{df}=1(P=0.14),\left.\right|^{2}=54.0 \% \\
\text { Test for overall effect: } Z=0.77(P=0.44)\end{array}$} \\
\hline
\end{tabular}

Favours treatment Favours control

Mean = media $; \mathrm{SD}=$ desvio padrão $; \mathrm{WMD}=$ diferença media ponderada $; \mathrm{CI}=$ interval de confiância .

\section{DISCUSSÃO}

No geral, a qualidade metodológica dos estudos foi considerada incerta ${ }^{23-29}$, quase todos os estudos incluídos foram classificados como 'baixo risco' em relação ao erro sistemático de risco de viés. No entanto, apenas dois estudos ${ }^{25,29}$ especificaram o mascaramento da avaliação do resultado. Seis estudos forneceram dados suficientes para realizar metanálise.

A maioria dos artigos incluídos apresentou uma tendência favorável à diminuição do IMC e diminuição circunferência da cintura no grupo que realizou o programa 
avaliado. No entanto, quando analisados em conjunto na metanálise, a diminuição do IMC não foi significante. O fato das pesquisas serem estudos piloto com amostra pequeno e de curta duração forneceu um poder insuficiente para testar diferenças entre os grupos nas mudanças no IMC, 0 que seria o resultado primário em um estudo em larga escala. Apenas um estudo avaliou uma intervenção de longo prazo (24 meses), mas não pode ser incluído na metanálise e não apresentou diferenças entre os dois grupos para IMC e CC ${ }^{29}$.

Em relação aos resultados subjetivos mencionados nos artigos, o conhecimento sobre hábitos saudáveis trouxe impacto em curto prazo, causado pela motivação gerada pelo programa. A maioria dos artigos relatou aumento da atividade física, redução do tempo em frente à televisão, diminuição do estilo de vida sedentário ou modificação da dieta (aumento do consumo de alimentos e água saudáveis, redução do consumo de bebidas açucaradas).

A intervenção melhorou o conhecimento sobre os benefícios da atividade física como uma mudança de atitude em relação à inatividade física, mas poucos estudos demonstraram a real perda de peso. Apenas um artigo cujo desfecho medido foi a pressão arterial ${ }^{28}$. Outras variáveis como colesterol, triglicerídeos, glicose e risco de doença cardiovascular foram abordados apenas em um estudo 25 .

Quando os pais são convidados a participar do programa e estão de olho na criança há incentivo para praticar o que foi aprendido na escola ou em qualquer outro 
local, contribuindo para a perda de peso ${ }^{23-27}$. Quando o programa foi de responsabilidade somente da criança, a motivação perdeu sua força.

Se os pais não compreenderem e encorajarem mudanças nos hábitos dos filhos, inevitavelmente isso não acontecerá, porque essa medida direta também exige mudanças nos hábitos dos pais (cuidadores ou familiares), organização do tempo de trabalho e lazer, bem como um local adequado para atividades físicas.

Estudos experimentais em humanos que investigaram os efeitos da duração do sono na obesidade são limitados. Parece não haver estudos experimentais que demonstrem que o ganho de peso é causado pela modificação da duração do sono. Também não existem estudos clínicos experimentais que demonstrem que a perda ou estabilização do peso em pessoas com sobrepeso ou obesidade pode ser alcançada por meio da modificação da duração do sono ${ }^{30}$.

Nesta revisão, foram encontrados dois estudos de intervenção em crianças para testar a hipótese de que a modificação da duração do sono impediria a obesidade infantil, mas eles não atendiam aos critérios de inclusão de idade. Foi encontrado um estudo controlado randomizado sobre intervenções relacionadas ao sono, alimentação e atividade para prevenir 0 excesso de peso desde 0 nascimento, embora este fosse protocolo ${ }^{12}$ e um estudo piloto randomizado foi projetado para aumentar a duração do sono na primeira infância, ensinando aos pais 
estratégias alternativas para acalmar as crianças a fim de reduzir a alimentação como primeira resposta à agitação ${ }^{31}$.

Para crianças, a prevenção e o controle da obesidade associados à duração do sono não foram explorados extensivamente. Além disso, e mais importante, as avaliações se basearam principalmente em medidas subjetivas da duração do sono. O TRS, incluindo ronco e AOS, também é uma condição que não pode ser negligenciada e precisa ser considerada entre as intervenções para o controle da obesidade infantil.

Este estudo mostrou que são necessárias estratégias escolares para a prevenção da obesidade, com base nas necessidades das crianças, e que se inclua a família. A prevenção da obesidade deve incluir um componente de promoção do sono, e isso pode ser mais benéfico para crianças de baixo nível socioeconômico e/ou de contextos socialmente desfavorecidos.

Ensaios sobre intervenções para prevenção e controle da obesidade precisam ter amostras adequadas; desenhos paralelos randomizados controlados duplo-cego com medidas de resultados clinicamente relevantes, além do IMC e circunferência da cintura, além de escalas validadas internacionalmente que sejam definidas por um consenso. Ensaios controlados randomizados bem projetados são necessários para avaliar a eficácia das intervenções na prevenção e controle da obesidade, incluindo atividade física, alimentação saudável e principalmente a qualidade do sono. 


\section{CONCLUSÕES}

O Programa GEMS - 12 semanas possibilitou diminuição da Circunferência da Cintura, com uma fraca qualidade de evidência. Há necessidade de mais ensaios clínicos randomizados para estratégias escolares de prevenção da obesidade, com base nas necessidades das crianças, incluindo a família e o sono, com ampliação dos desfechos estudados e tamanho da amostra.

\section{AGRADECIMENTOS}

Conselho Nacional de Desenvolvimento Científico e Tecnológico - CNPq, Edital \#559187/2009-2.

\section{REFERÊNCIAS}

1. Ochiai H, Shirasawa T, Shimada N, Ohtsu T, Nishimura R, Morimoto $A$, et al. Sleep Duration and Overweight among Elementary Schoolchildren: A Population-based Study in Japan. Acta Med Okayama 2012;66:93-9. https://doi.org/10.18926/AMO/48259

2.Silva GAP, Balaban G, Motta MEF. Prevalência de sobrepeso e obesidade em crianças e adolescentes de diferentes condições sócioeconômicas. Rev Bras Saúde Matern Infant 2005;5:53-9.

http://dx.doi.org/10.1590/S1519-38292005000100007

3.Van Cauter E, Knutson KL. Sleep and the epidemic of obesity in children and adults. Eur J Endocrinol 2008;159:5966. https://doi.org/10.1530/EJE-08-0298

4.Sabanayagam C, Zhang R, Shankar A. Markers of Sleep-Disordered Breathing and Metabolic Syndrome in a Multiethnic Sample of US Adults: Results from the National Health and Nutrition Examination Survey 2005-2008. Cardiol Res Pract 2012;2012:630802. https://doi.org/10.1155/2012/630802

5.Valbuza JS, de Oliveira MM, Conti CF, Prado LB, de Carvalho LB, do Prado GF. Methods for increasing upper airway muscle tonus in treating obstructive sleep apnea: systematic review. Sleep Breath 2010;14:299-305. https://doi.org/10.1007/s11325-010-0377-9 
6. Carvalho LBC, Prado LBF, Silva L, de Almeida MM, Almeida e Silva $\mathrm{T}$, Lora MI, et al. Cognitive dysfunction in children with sleepdisordered breathing. J Children Neurol 2005;20:400-04.

https://doi.org/10.1177/08830738050200050101

7.Beebe DW, Miller N, Kirk S, Daniels SR, Amin R. The association between obstructive sleep apnea and dietary choices among obese individuals during middle to late childhood. Sleep Med 2011;12:79799. https://doi.org/10.1016/j.sleep.2010.12.020

8.Zheng $\mathrm{H}$, Berthoud $\mathrm{H}-\mathrm{R}$. Neural systems controlling the drive to eat: mind versus metabolism. Physiology 2008;23:75-83.

https://doi.org/10.1152/physiol.00047.2007

9. Carvalho LB, Prado LB, Ferreira VR, da Rocha Figueiredo MB, Jung $A$, de Morais JF, et al. Symptoms of sleep disorders and objective academic performance. Sleep Med 2013;14:872-6.

https://doi.org/10.1016/j.sleep.2013.05.011

10.Nixon GM, Thompson JM, Han DY, Becroft DM, Clark PM, Robinson $\mathrm{E}$, et al. Short sleep duration in middle childhood: risk factors and consequences. Sleep 2008;31:71-8.

https://doi.org/10.1093/sleep/31.1.71

11.Spruyt K, Molfese DL, Gozal D. Sleep Duration, Sleep Regularity, Body Weight, and Metabolic Homeostasis in School-aged Children. Pediatrics 2011;127:345-52. https://doi.org/10.1542/peds.20100497

12.Cappuccio FP, Taggart FM, Kandala N, Currie A, Peile E, Stranges $S$, et al. Meta-Analysis of Short Sleep Duration and Obesity in Children and Adults. Sleep 2008;31:619-26.

https://doi.org/10.1093/sleep/31.5.619

13.Taylor BJ, Heath AL, Galland BC, Gray AR, Lawrence JA, Sayers RM, et al. Prevention of Overweight in Infancy (POI.nz) study: a randomized controlled trial of sleep, food and activity interventions for preventing overweight from birth. BMC Public Health 2011;19:942. https://doi.org/10.1186/1471-2458-11-942

14.Touchete E, Petit D, Tremblay RE, Boivin M, Falissard B, Genolini $C$, et al. Associations between sleep duration patterns and overweight/obesity at age 6. Sleep 2008;31:1507-14. https://doi.org/10.1093/sleep/31.11.1507

15. Moraeus L, Lissner L, Yngve A, Poortvliet E, Al-Ansari U, Sjöberg A. Multi-level influences on childhood obesity in Sweden: societal factors, parental determinants and child's lifestyle. Int J Obes (Lond) 2012;36:969-76. https://doi.org/10.1038/ijo.2012.79

16.Dündar $\mathrm{C}, \mathrm{Oz} \mathrm{H}$. Obesity-related factors in Turkish school children. Sci World J 2012;2012:353485.

https://doi.org/10.1100/2012/353485

17.Campos LF, Gomes JM, Oliveira JC. Obesidade Infantil, Atividade Física e Sedentarismo em crianças do 10 ciclo do ensino básico da cidade de Bragança (6 a 9 anos). Motric 2008;4:17-24.

18. Lanningham-Foster $L$, Jensen $T B$, Foster RC, Redmond $A B$, Walker $B A$, Heinz $D$, et al. Energy expenditure of sedentary screen time 
compared with active screen time for children. Pediatrics 2006;118:e1831-5. https://doi.org/10.1542/peds.2006-1087

19.Robinson TN. Reducing children's television viewing to prevent obesity: a randomized controlled trial. JAMA 1999;282:1561-7. https://doi.org/10.1001/jama.282.16.1561

20.McCall A, Raj R. Exercise for Prevention of Obesity and Diabetes in Children and Adolescents. Clin Sports Med 2009;28:393-421. https://doi.org/10.1016/j.csm.2009.03.001

21.DerSimonian R, Laird N. Meta-analysis in clinical trials. Controlled Clin Trials 1986;7:177-88. https://doi.org/10.1016/01972456(86)90046-2

22. Higgins JPT, Thompson SG, Deeks JJ, Altman DG. Measuring inconsistency in meta-analyses. BMJ 2003;327:557-60. https://doi.org/10.1136/bmj.327.7414.557

23.Baranowski T, Baranowski JC, Cullen KW, Thompson DI, Nicklas T, Zakeri IE, et al. The Fun, Food, and Fitness Project (FFFP): the Baylor GEMS pilot study. Ethn Dis 2003;13(Suppl 1):S30-9.

24. Beech BM, Klesges RC, Kumanyika SK, Murray DM, Klesges L, McClanahan B, et al. Child- and parent-targeted interventions: the Memphis GEMS pilot study. Ethn Dis 2003;13(Suppl 1):S40-53.

25.Robinson TN, Killen JD, Kraemer HC, Wilson DM, Matheson $\mathrm{DM}$, Haskell $\mathrm{WL}$, et al. Dance and reducing television viewing to prevent weight gain in African-American girls: the Stanford GEMS pilot study. Ethn Dis 2003;13(Suppl 1):S65-77.

26.Story M, Sherwood NE, Himes JH, Davis M, Jacobs DR Jr, Cartwright $Y$, et al. An after-school obesity prevention program for African-American girls: the Minnesota GEMS pilot study. Ethn Dis 2003;13(Suppl 1):S54-64.

27.Kalavainen MP, Korppi MO, Nuutinen OM. Clinical efficacy of group-based treatment for childhood obesity compared with routinely given individual counseling. Int J Obes (Lond) 2007;31:1500-8. https://doi.org/10.1038/sj.ijo.0803628

28.Chen JL, Weiss S, Heyman MB, Vittinghoff E, Lustig R. Pilot study of an individually tailored educational program by mail to promote healthy weight in Chinese American children. J Spec Pediatr Nurs 2008;13:212-22. https://doi.org/10.1111/j.1744-6155.2008.00155.x 29.Klesges RC, Obarzanek E, Kumanyika S, Murray DM, Klesges LM, Relyea GE, et al. The Memphis Girls' health Enrichment Multi-site Studies (GEMS): an evaluation of the efficacy of a 2-year obesity prevention program in African American girls. Arch Pediatr Adolesc Med 2010;164:1007-14.

https://doi.org/10.1001/archpediatrics.2010.196

30.Gozal D, Kheirandish-Gozal L. Childhood obesity and sleep: relatives, partners, or both? A critical perspective on the evidence. Ann N Y Acad Sci 2012;1264:135-41. https://doi.org/10.1111/j.17496632.2012.06723.x

31.Paul IM, Savage JS, Anzman SL, Beiler JS, Marini ME, Stokes JL, et al. Preventing obesity during infancy: a pilot study. Obesity (Silver Spring) 2011;19:353-61. https://doi.org/10.1038/oby.2010.182 